\title{
Disciplina interprofissional em saúde: avaliação de discentes de Odontologia
}

\author{
Amanda Meira Saraiva*; Isabela Regina Grilo Silva**; Luiz Fernando Lolli**; Mitsue Fujimaki**; \\ Rozilda das Neves Alves***; Edson Roberto Arpini Miguel****; Nadeje da Rocha Mialchi*****; \\ Najara Barbosa da Rocha**
}

\author{
* Programa de Residência em Odontologia em Saúde Coletiva e da \\ Família, Departamento de Odontologia, UEM \\ ** Departamento de Odontologia, UEM \\ *** Departamento de Psicologia, UEM \\ **** Departamento de Medicina, UEM \\ ***** Universidade Estadual de Campinas
}

Recebido em 12/01/2018. Aprovado em 22/09/2018.

\begin{abstract}
RESUMO
A educação interprofissional é uma estratégia pedagógica na qual duas ou mais profissões trabalham e aprendem juntas, priorizando a integração, com a finalidade de mudar o perfil profissional na área da saúde e melhorar a qualidade do cuidado e resolutividade na atenção primária. A disciplina Atenção em Saúde é uma proposta interprofissional, desenvolvida em sete cursos da área da saúde na Universidade Estadual de Maringá, oportunizando ao aluno vivência no Sistema Único de Saúde (SUS), utilizando a problematização de situações em equipe. Objetivou-se apresentar a avaliação dos estudantes de Odontologia sobre a disciplina Atenção em Saúde, por meio da análise das narrativas de seus portfólios avaliativos. Trata-se de um estudo exploratório e descritivo, com análise documental como técnica de investigação, com amostragem até saturação dos dados. A análise dos documentos permitiu mostrar que os acadêmicos ressaltam a importância do trabalho interprofissional, beneficiando trabalhadores e pacientes e a relevância do cirurgião-dentista na equipe de saúde. Constatou-se o conhecimento experienciado do aluno sobre o SUS, capaz de destacar falhas e qualidades, bem como o reconhecimento sobre a eficácia do modelo ativo de aprendizagem, para capacitá-los a enfrentar positivamente as adversidades. Conclui-se que os acadêmicos apontaram as práticas colaborativas interprofissionais e a utilização de metodologias ativas de ensinoaprendizagem como estratégias positivas para formação qualificada, estando em consonância com as Diretrizes Curriculares Nacionais de Odontologia quanto à formação profissional para o trabalho em equipe e compreensão das reais necessidades de saúde da população.
\end{abstract}

Descritores: Odontologia. Educação Superior. Saúde. Aprendizagem Baseada em Problemas. Avaliação. 


\section{INTRODUÇÃO}

Atualmente, vivencia-se um momento em que o paradigma da organização do trabalho, baseado na extrema segmentação da proposta flexneriana é inadequado. Este modelo favorece uma formação acrítica e restrita aos profissionais, sendo pouco resolutiva, além de não capacitar para lidar com os reais problemas de saúde da população ${ }^{1}$. Assim, a mudança na formação profissional de estudantes e professores do campo da saúde se torna cada vez mais necessária, porém é possível destacar que esta ainda permanece como um desafio ${ }^{2}$.

A importância do trabalho em equipe nos serviços de saúde deve ser ressaltada, principalmente, pelo aspecto de integralidade $\mathrm{e}$ resolutividade nos cuidados de saúde ${ }^{2}$. A abordagem integral dos pacientes é facilitada pela soma de olhares dos distintos profissionais que compõem as equipes interdisciplinares. Dessa maneira, há possibilidade de obter maior impacto sobre os diferentes fatores que interferem no processo saúde-doença ${ }^{2}$ e maior eficiência e eficácia no serviço de saúde ${ }^{3}$.

A educação interprofissional é uma estratégia pedagógica na qual duas ou mais profissões trabalham e aprendem juntas, priorizando o trabalho em equipe, integração e troca de conhecimento ${ }^{4}$ e ocorre quando os estudantes de duas ou mais profissões aprendem sobre os outros, com os outros e entre si para possibilitar a efetiva colaboração e melhorar os resultados na saúde ${ }^{5}$. A interprofissionalidade ainda é um grande desafio, ela ajuda a melhorar a qualidade do cuidado e resolutividade na atenção primária e esta experiência tem mostrado mudanças importantes no perfil do profissional formado $^{6,7}$.

A atividade entre várias profissões promove experiências necessárias para a formação de uma força de trabalho de saúde colaborativa preparada para a prática ${ }^{8,9}$. Assim, esses profissionais aperfeiçoam as habilidades e compartilham o gerenciamento dos casos entre os membros dessa equipe em prol de um atendimento de melhor qualidade e de acordo com as reais necessidades da população.

É necessário enfatizar que a busca da compreensão do processo saúde-doença de forma não fragmentada é essencial, devendo levar em conta as necessidades dos usuários ${ }^{10}$. Esta preocupação com a formação do perfil profissional adequado que atenda de forma integral as necessidades de saúde da população tem sido a busca do curso de Odontologia da Universidade Estadual de Maringá (UEM), que a partir de 1992 iniciou mudanças consistentes para aderir à proposta de um currículo integrado, já em concordância com as Diretrizes Curriculares Nacionais (DCN) para os cursos de graduação em Odontologia aprovadas dez anos depois $^{11,12}$. Em 2005, teve o Programa Nacional de Reorientação da Formação Profissional em Saúde (Pró-Saúde) aprovado e em 2009, iniciou a abordagem integradora e tutorial com a participação no Programa de Educação pelo Trabalho para a Saúde (PET-Saúde), que consiste em uma aprendizagem ativa com análise crítica das necessidades sociais e os princípios e diretrizes do Sistema Único de Saúde (SUS) levando grupos pequenos de alunos a vivenciarem na prática a inter e a multiprofissionalidade, a interdisciplinaridade e a integralidade no cuidado à saúde ${ }^{13,14}$.

As experiências de integração entre os cursos da área da saúde desde o projeto Prósaúde I e os subsequentes editais para a reorientação da formação profissional na UEM possibilitaram a elaboração da disciplina Atenção em Saúde, que contempla o trabalho interprofissional no primeiro ano em sete cursos da área da saúde (Medicina, Farmácia, Biomedicina, Educação Física, Psicologia, Enfermagem e Odontologia), iniciada no 
primeiro semestre do ano de $2015^{15}$.

A disciplina Atenção em Saúde utiliza metodologias ativas de ensino e aprendizagem, em consonância com as DCN dos cursos da área da saúde e insere o aluno no serviço público de saúde logo no primeiro ano de graduação. A aprendizagem e a busca pelo conhecimento são responsabilidades do estudante, sendo o professor um facilitador, visando dar autonomia ao discente na tomada de decisões do cotidiano do trabalho ${ }^{15}$. Essa abordagem pedagógica é importante para a mudança no processo pelo qual a assistência à saúde é produzida ${ }^{1}$.

Esta proposta é pioneira e inovadora no país, poucas universidades ${ }^{1,16}$ tem utilizado a abordagem interprofissional como disciplina curricular $^{4,17}$, porém nenhuma de forma obrigatória entre os cursos de graduação na área da saúde. Portanto, é importante a constante avaliação das percepções e do ensino dos futuros profissionais de saúde. Assim, este estudo tem como objetivo apresentar a avaliação de estudantes de Odontologia sobre a disciplina de Atenção em Saúde, por meio da análise das narrativas dos portfólios avaliativos.

\section{METODOLOGIA}

Trata-se de um estudo exploratório e descritivo, com abordagem qualitativa, utilizando a análise documental como técnica de investigação, baseando-se em portfólios individuais construídos ao longo do semestre letivo pelos acadêmicos do primeiro ano de graduação do curso de Odontologia da UEM, cursando a disciplina Atenção em Saúde.

\section{Educação interprofissional}

A disciplina Atenção em Saúde é uma disciplina que ocorre no primeiro ano de graduação de sete cursos da saúde, com o total de 68 horas, realizada no município de Maringá, Estado do Paraná. Sua base está no conhecimento e na análise do território dos serviços de saúde, por meio da vivência do trabalho interprofissional e interdisciplinar em diversos cenários do SUS. O objetivo da disciplina é a organização e dinâmica de funcionamento dos serviços no SUS, por meio da compreensão das ações desempenhadas sobre educação, promoção e recuperação da saúde; na prevenção de doenças e melhoria da qualidade de vida e do conhecimento de saúde da população. É desenvolvida por meio de ciclos de problematização, baseados no Arco de Maguerez, que parte da observação da realidade, a identificação de pontos chave para o problema, a teorização, o levantamento de hipótese de solução, a elaboração de propostas para intervenção e discussão de soluções para temas e problemas encontrados ${ }^{18}$. Ocorre nas Unidades Básicas de Saúde (UBS) e os estudantes são distribuídos em grupos multiprofissionais de 10 a 11 alunos, orientados por um tutor (docente) e auxiliados por um preceptor (profissional do serviço de saúde). A avaliação do graduando é centrada na mensuração das competências do eixo cognitivo, demonstradas na construção de um portfólio, além de avaliações diárias dos tutores, a partir das competências desenvolvidas no eixo psicomotor e afetivo. Ocorrem também uma avaliação por parte dos próprios alunos sobre os colegas (avaliação aos pares) e uma autoavaliação centrada em alguns eixos centralizadores da disciplina.

O portfólio oportuniza que os estudantes aprendam melhor e de forma mais integral, devido à reflexão necessária para sua elaboração, que exige do aluno mais atenção e compromisso com as atividades ${ }^{19}$. A construção de portfólios permitiu reunir informações suficientes para análise da evolução dos alunos na disciplina, envolvendo a questão da nova metodologia de ensino empregada ${ }^{20}$ e o contato do aluno com o SUS, estimulando assim a autonomia e o 
pensamento crítico-reflexivo $^{21}$. Esses documentos contêm narrativas dos acadêmicos em relação às suas atividades semanais, pesquisas efetuadas no decorrer da disciplina e também algumas questões com objetivo de identificar as opiniões, percepções e apreensões dos alunos em relação à disciplina e a metodologia empregada na mesma.

\section{Amostragem}

O aprofundamento e a compreensão do tema foram considerados com maior importância, devido ao fato do estudo ser de natureza qualitativa. A amostragem foi intencional e a coleta de dados foi interrompida quando constatado que elementos novos, para subsidiar a teorização, não foram mais encontrados, totalizando sete portfólios analisados.

\section{Coleta e análise de dados}

Os textos dos portfólios foram transcritos na íntegra e analisados pela técnica qualitativa de Análise de Conteúdo, preconizada por Bardin ${ }^{22}$. Foram realizadas a categorização das respostas e análise em profundidade dos conteúdos manifestos pelos atores sociais envolvidos. Nesta investigação, foram encontrados quatro temas centrais: a experiência da educação interprofissional; o uso de metodologias ativas; o contato com o SUS; e a postura do estudante em relação à disciplina considerando as DCN na área da saúde.

\section{RESULTADOS E DISCUSSÃO}

A disciplina Atenção em Saúde da UEM vem obtendo resultados positivos na avaliação realizada pelos discentes. É possível destacar, por meio das opiniões dos próprios alunos, o quão enriquecedora é para os estudantes. A seguir são apontados e discutidos os temas centrais resultantes da análise documental.

\section{Experiência da educação interprofissional}

Muitos sistemas de saúde no mundo encontram-se fragmentados e com dificuldades para gerenciar as necessidades de saúde não atendidas da população. $\mathrm{O}$ ensino interprofissional vem para suprir esta lacuna nos serviços de saúde, porém é possível notar que no Brasil ainda há poucas experiências relacionadas a propostas interprofissionais, o que também se nota em relação a pesquisas na literatura científica, as quais são escassas ${ }^{16}$. Poucos cursos têm utilizado a educação interprofissional em suas grades curriculares ${ }^{4}$. Algumas instituições fazem uso de disciplinas interprofissionais de maneira facultativa, de forma que uma grande parte dos alunos não vivencie esta oportunidade $^{19}$. Nota-se ainda mais escassez em estudos abordando experiências integradoras e interprofissionais de maneira não elegível, quando o foco é o curso de Odontologia ${ }^{15}$.

É evidente que com a experiência interprofissional os alunos aprendem habilidades necessárias para se tornarem parte da força de trabalho de saúde em equipe. Dessa forma, quando o estudante se apropria dos pressupostos da educação interprofissional, na qual a prática colaborativa melhora o resultado na saúde ${ }^{23}$, é capaz de entrar no local de trabalho e aprender sobre os outros e com os outros para possibilitar a efetiva colaboração e melhorar os resultados na saúde, modificando a prática e a assistência ao paciente $^{24}$. Além disso, a educação interprofissional prioriza a integração e a flexibilidade da força de trabalho, reconhecendo e respeitando às especificidades de cada profissão ${ }^{4}$, sendo que esta prática se faz essencial para integralidade do cuidado em saúde ${ }^{17}$. Este trabalho em equipe de forma colaborativa foi evidenciado neste estudo, pois a maior parte dos alunos acredita que o trabalho interprofissional é enriquecedor pela possibilidade de aprender com outras áreas. Algumas falas analisadas foram 
relacionadas aos benefícios dessa experiência:

(A2) "preparados para a prática interprofissional (...) buscando integração por meio da realização de ações de promoção, proteção $e$ prevenção de agravos a saúde."

(A4) "quando se trabalha em equipe o serviço é realizado com maior eficácia" em que há uma maior (A5) "força de trabalho de saúde "colaborativa preparada para a prática."

Além disso, foi citado que a educação interprofissional pode formar profissionais melhores capacitados:

(A1) "grande desenvolvimento tanto profissional quanto pessoal."

A atuação interprofissional se faz presente nesta disciplina e aparece na análise dos alunos, ou seja, a necessidade do perfil mais humanista e ético, como a que segue:

(A1) "principalmente que saiba respeitar as diferenças, e respeitar todos os profissionais e cada uma de suas especialidades, para em conjunto adquirir um resultado benéfico para todos."

\section{O uso de metodologias ativas}

Há muito tempo, profissionais de saúde vêm recebendo formação baseada na metodologia de ensino-aprendizagem tradicional, em que o professor é o sujeito ativo no processo, repassando seu conhecimento aos alunos por meio de aula expositiva ${ }^{25}$, sofrendo forte influência do mecanicismo de inspiração cartesiana e fragmentada. Esta metodologia pode restringir o processo de ensino à reprodução do conhecimento, no qual o educador detém o monopólio do poder, assumindo o papel de transmissor de conteúdos e formulador de questões, ao passo que, o educando precisa dar as respostas, reter e repetir conteúdos, sem a necessária crítica e reflexão imprescindíveis à uma aprendizagem significativa ${ }^{26}$.

$\mathrm{Na}$ saúde, as pedagogias ativas também não tratam de uma novidade, pois a Medicina Preventiva, desde 1950, tem defendido a readequação curricular e a incorporação de metodologias ativas na formação profissional. A utilização destes métodos de ensinoaprendizagem parece ser eficiente para a formação de um profissional da saúde mais crítico e capaz de enfrentar os desafios postos pela realidade social ${ }^{1}$.

A utilização de metodologias ativas de ensino-aprendizagem, no qual o aluno é o protagonista do seu próprio processo de formação, tem demonstrado grande sucesso ${ }^{6}$. Possuem como base, estratégias de ensino fundamentadas na concepção pedagógica críticoreflexiva que permitem uma leitura e intervenção da realidade com a interação entre os diversos atores e valorização da construção coletiva do conhecimento por seus diferentes saberes e cenários de aprendizagem. É capaz de integrar a subjetividade no processo educativo, estimulando a reflexão sobre a realidade em que se inserem os alunos ${ }^{27}$. Também são práticas que estimulam a criatividade na construção de soluções aos problemas e que promovem a liberdade no processo de pensar e de agir estimulando assim, o processo de autonomia do aluno $^{26}$.

A proposta de problematização da aprendizagem, do Arco de Maguerez, está baseada nos princípios de Pedagogia Ativa, em que o professor deve ser um facilitador com função de organizar o grupo para que busque informações, conhecimentos, avaliados como necessários para resolver dado problema ${ }^{1}$.

A análise permitiu verificar a importância que os estudantes deram aos benefícios dessa experiência; todos os alunos concordam que 
metodologias ativas de ensino formam um profissional melhor preparado para a prática, postulando maior humanização, visão ampliada no meio profissional e multiprofissional. Os benefícios ao futuro profissional apontados pelos estudantes foram:

(A1) "é preparado para lidar com possíveis problemas que irá encontrar e assim já terá maior experiência sobre isso."

(A2) "tendo como objetivo o bem comum" e enfatiza a questão do ganho de experiência, dizendo que (A6) "quando temos a experiência, o ganho de aprendizagem é muito maior do que simplesmente pela transmissão de informação."

A mudança de foco do processo de educação visa a autonomia, criatividade $\mathrm{e}$ responsabilidade do estudante, possibilitando que o aluno aprenda a buscar soluções e a resolver problemas profissionais por si mesmo ${ }^{26}$.

Mesmo com opiniões satisfatórias sobre a utilização das metodologias ativas de aprendizagem, é possível encontrar na literatura críticas a esse método, já que os alunos podem se sentir perdidos na busca do conhecimento, existindo uma espécie de lacuna no saber ideal e também a presença de relatórios exigidos como complemento, que geralmente são extensos e cansativos $^{28}$, além de gerar certa insegurança aos estudantes e requerer um grande esforço dos mesmos no processo, exigindo muitas vezes uma mudança abrupta de comportamento, maturidade e organização $^{29}$. Esse descontentamento foi demonstrado por um dos alunos: (A3) "um tanto quanto cansativo fazer relatórios e pesquisas semanais quando se faz um curso integral." demandando maior tempo de estudos que o método até então utilizado. Assim, é importante que o facilitador dê feedback avaliativo tão logo perceba que algum estudante apresente dificuldade ou descontentamento na disciplina, por meio das avaliações diárias realizadas, estimulando constantemente seu processo de ensino-aprendizagem.

\section{Contato com o SUS}

A interligação entre ensino-serviçocomunidade e a utilização de metodologias ativas de ensino-aprendizagem são evidenciadas como estratégias para a formação de profissionais voltados para o mercado de trabalho e para as necessidades reais da população ${ }^{5}$ exigindo que o aluno obtenha uma preparação para atuar no serviço público de saúde, sendo, consequentemente, melhor formado e preparado para a realidade da população.

Alguns alunos relataram esta inserção no SUS como algo inovador, em que foi traduzido como (A3) "animador" o contato com o sistema único de saúde, principalmente pelo ponto de vista profissional, além de ser definido como (A4) "experiência diferente e ideia interessante" esse aprofundamento em um sistema que alguns nunca tinham tido contato anterior.

Notou-se ainda uma visão mais crítica sobre os fatores que impossibilitam a funcionalidade ideal do sistema público de saúde, devido ao pensamento econômico dos gastos mínimos com a questão social, o que dificulta que o ideal previsto da Reforma Sanitária Brasileira seja implementado, ou seja, um sistema de saúde único, integral, gratuito, equitativo e resolutivo para toda a população ${ }^{30}$, configurado nas perspectivas dos estudantes como:

(A3) "o descaso com a saúde por parte dos órgãos governamentais"

(A5) "que faltam recursos e estrutura para um atendimento pleno."

Outro fator importante descrito foram os destaques dado ao SUS como sistema de saúde público que pode atuar de forma permanente e de 
como está mais próximo das pessoas com valores humanos, solidários e, além de tudo, resolutivos $^{31}$. Este último apontamento visa desmontar a concepção que permeia o serviço público é culpado pelos problemas, em sua maioria, advindos do Estado ${ }^{32}$, para exemplificar transcrevemos a frase do aluno:

(A5) "esse conhecimento também me fez quebrar algumas teorias, como a de que os funcionários do sistema público não se importavam com os pacientes que atendiam."

Os discentes de Odontologia também apontam pontos positivos, principalmente no campo dos trabalhadores do setor público. Nos últimos anos houve uma nova atribuição aos profissionais de saúde que passaram a ser considerados como agentes de mudança no contexto de atenção à família e ainda facilitadores no processo da educação em saúde $^{33}$. Foram apontados pelos alunos como:

(A3) "profissionais que trabalham na UBS visitada extremamente capacitados, ética na forma de trabalho, apesar da dificuldade são exemplos de profissionais."

(A7) "todos os funcionários foram sempre gentis e dispostos a tirar dúvidas."

Após estas experiências os alunos se imaginaram como futuros profissionais neste contexto.

(A3) "vamos conhecer muitos problemas e muitas dificuldades que precisam ser superadas pelos profissionais da saúde diariamente."

A postura do estudante em relação à disciplina, considerando as DCN na área da saúde

As DCN do curso de graduação em Odontologia preconizam o perfil do cirurgiãodentista baseado em uma formação generalista, humanista, crítica e reflexiva, para atuar em todos os níveis de atenção à saúde, com base no rigor técnico e científico ${ }^{5}$. Também orientam que as avaliações dos alunos sejam formativas e se baseiem nas competências, habilidades, atitudes e conteúdos curriculares, utilizando metodologias e critérios para o acompanhamento e avaliação do processo ensino-aprendizagem do próprio curso $^{26}$. Pela análise da avaliação dos alunos nos portfólios, sugerem que a postura do estudante ocorre a partir do aproveitamento do método ativo utilizado e da atuação interprofissional, vivenciando, assim, o contato com a realidade para se tornar um profissional melhor, humanizado, ético, com humildade, aprendendo a (A2) "valorizar todos os profissionais e usuários da saúde de forma unificada."

Os relatos dos estudantes classificam essa disciplina como:

(A4) "de extrema importância para sua vida profissional como um todo" e que (A1) "a disciplina deve ser abordada com seriedade e respeito, para o melhor aproveitamento de uma oportunidade que não existe em todos os cursos."

A premissa colaborativa na educação interprofissional é um aspecto importante na formação profissional, pois prepara o profissional para melhor responder às necessidades de saúde locais. A integração ensino-serviço oportuniza experiências de aprendizagem significativas que tem resultado em melhorias nos serviços de saúde e na formação profissional. Nessa via, a utilização dessa metodologia, em que o professor assume o papel de facilitador e o aluno a responsabilidade pela busca do conhecimento, sendo percebido um grau maior de autonomia na tomada de decisões do cotidiano de trabalho.

A disciplina também teve entre seus pontos importantes a oportunidade de troca de 
conhecimentos ao colocar diversos alunos de diferentes cursos da área da saúde juntos.

(A4) "ao fazê-lo interagir com outros acadêmicos, ganhando conhecimentos que até então ele não tinha acesso."

Uma experiência semelhante ocorre na disciplina de "Práticas Integradas em Saúde I" (PIS I) da Universidade Federal do Rio Grande do Sul (UFRGS), implementada em 2012, integrando 13 cursos de graduação ${ }^{17}$. Diferente da disciplina descrita neste artigo, a experiência levada a cabo pela UFRGS, teve caráter eletivo para 10 de seus cursos, inclusive Odontologia, e adicional para os cursos de Medicina, Enfermagem e Saúde Coletiva. Ou seja, existem limites de vagas para seleção dos alunos de cada curso por semestre ${ }^{17}$. Entre ambas há duas diferenças. A primeira é que na UEM a disciplina é desenvolvida em todos os cursos integrados e a segunda consiste na forma de avaliação final, em que na PIS I é exigido a apresentação de um seminário ao final da disciplina, além da entrega do portfólio ${ }^{17}$.

Também é no contexto da educação interprofissional que se insere o desenho curricular dos novos cursos do Campus Baixada Santista da Universidade Federal de São Paulo, nos quais os alunos de todos os anos do curso vivenciam momentos de aprendizagem compartilhada (80\% no primeiro ano, $40 \%$ no segundo ano, $20 \%$ no terceiro ano e reuniões semanais no quarto ano) ${ }^{17}$.

Com relação à análise dos registros, verificou-se que os portfólios tiveram um perfil predominantemente narrativo a partir das atividades realizadas. Pode-se, por meio deles, inferir, que para as próximas turmas será necessário dar mais atenção ao desenvolvimento de uma visão mais crítica e reflexiva sobre as atividades realizadas pelos graduandos, resultando em portfólios que possam consubstanciar o processo de avaliação, que supere a visão do estudante e proporcione melhorar a construção de uma visão de saúde integrada e colaborativa ${ }^{21}$.
As limitações deste estudo devem ser ressaltadas. A primeira consiste na análise qualitativa. Estudos quantitativos longitudinais com amostras mais significativas devem ser realizados para complementar as informações e aumentar o nível de evidência do estudo. Outras pesquisas são necessárias para verificar o desempenho desta disciplina utilizando outros desenhos de estudo, tais como transversais ou longitudinais e com amostras representativas.

\section{CONCLUSÕES}

As avaliações dos estudantes mostraram a importância do trabalho em ambiente interprofissional, gerando benefícios aos trabalhadores e pacientes, bem como a importância do profissional da Odontologia dentro da equipe de saúde. Foi verificado conhecimento enriquecido do aluno sobre o SUS, com falhas e qualidades destacadas. Também houve o reconhecimento, por parte dos acadêmicos, da eficácia do modelo ativo de aprendizagem, sendo capaz de formar um profissional melhor capacitado e preparado para enfrentar adversidades de maneira positiva no ambiente de trabalho. As dificuldades apontadas pelos graduandos foram relacionadas à falta de recursos materiais, financeiros e humanos nos serviços públicos de saúde.

Os resultados também apontaram que os objetivos da disciplina (promover educação interprofissional, promoção de práticas colaborativas em saúde) foram alcançados, sendo a troca de conhecimentos entre alunos de diversos cursos da saúde essencial para o crescimento do indivíduo e do grupo.

\section{ABSTRACT \\ Interprofessional discipline in health: assessment of Dentistry students \\ Interprofessional education is a pedagogical strategy in which two or more professions work and learn together, prioritizing integration in order to change the professional profile of health,}


and improve the quality of care and resolution in primary care. The Health Care discipline is an interprofessional proposal, developed in seven courses at the State University of Maringá, giving students from the Unified Health System (UHS), the opportunity to learn how to use the problematization of team situations. The aim was to present the perception of Dentistry students of the Attention in Health discipline, through analysis of the narratives in the evaluation portfolio. This was an exploratory and descriptive study using document analysis as a research technique, in which sampling continued until data saturation was reached. Analysis of the portfolios allowed the authors to show that academic students emphasized the importance of interprofessional work, its benefit to workers and patients, and the relevance of including dentists in the health teams. The students' knowledge about Unified Health System was able to highlight flaws and qualities, and recognize the effectiveness of the active learning model in enabling them to face adversities positively. It was concluded that the academic students pointed out that interprofessional collaborative practices and the use of active learning methodologies were positive strategies for a qualified education and training, and were in agreement with the curricular guidelines of the Dentistry course for professional education in and understanding of team work within the context of the real health needs of the population. Descriptors: Dentistry. Education, Higher. Health. Problem-Based Learning. Evaluation.

\section{REFERÊNCIAS}

1. Conterno SFR, Lopes RE. Inovações do século passado: origens dos referenciais pedagógicos na formação profissional em saúde. Rev Trab Educ Saúde. 2013;11(3):50323.

2. Araújo MBS, Rocha PM. Trabalho em equipe: um desafio para a consolidação da estratégia de saúde da família. Ciênc Saúde Coletiva. 2007;12(2):455-64.

3. Peduzzi M, Carvalho BG, Mandú ENT, Souza
GC, Silva JAM. Trabalho em equipe na perspectiva da gerência de serviços de saúde: instrumentos para a construção da prática interprofissional. Physis. 2011;21(2):629-46.

4. Aguilar-da-Silva RH, Scapin LT, Batista NA. Avaliação da formação interprofissional no ensino superior em saúde: aspectos da colaboração e do trabalho em equipe. Avaliação (Campinas). 2011;16(1):165-84.

5. Carvalho WM, Cawahisa PT, Scheibel PC, Botelho JN, Terada RSS, Rocha NB, et al. Aceitação da utilização de metodologias ativas nos estágios no SUS por discentes da graduação e pós-graduação em Odontologia. Rev ABENO. 2016;16(1):88-98.

6. Goelen G, de Clercq G, Huyghens L, Kerckhofs E. Measuring the effect of interprofessional problem-based learning on the attitudes of undergraduate health care students. Med Educ J. 2006;40(6):555-61.

7. Hind M, Norman I, Cooper S, Gill E, Hilton $\mathrm{R}$, Judd $\mathrm{P}$, Jones $\mathrm{S}$. Interprofessional perceptions of health care students. J Interprof Care. 2003;17(1):21-34.

8. Organização Mundial de Saúde. Marco para Ação em Educação Interprofissional e Prática Colaborativa. Genebra: OMS. 2010. [Acesso em: 08 fev. 2017]. Disponível em: http://www.sbfa.org.br/fnepas/oms traduzido 2010.pdf.

9. McNair R, Stone N, Sims J, Curtis C. Australian evidence for interprofessional education contributing to effective team work preparation and interest in rural practice. $\mathrm{J}$ Interprof Care. 2005;19(6):579-94.

10. Chesani FH, Maestrelli SRP, Cutolo LRA, Nunes R. Aprendizagem baseada em problemas e a formação do fisioterapeuta: estudo de caso. Rev Trab Educ Saúde. 2017;15(3):931-50.

11. Terada RSS, Nakama L. A implantação das Diretrizes Curriculares Nacionais de 
Odontologia: a experiência de Maringá. São Paulo: Hucitec. 2004.

12. Brasil. Ministério da Educação. Conselho Nacional de Educação. Câmara de Educação Superior. Institui Diretrizes Curriculares Nacionais do curso de graduação em Odontologia, Brasília, DF, 19 fev. 2002. [Acesso em: 09 fev. 2017]. Disponível em: http://portal.mec.gov.br/cne/arquivos/pdf/CE S032002.pdf.

13. Terada RSS, Hayacibara MF, Rigolon CJ, Silva MC, Lolli LF, Hidalgo MM. Implementação do Pró-Saúde no Curso de Odontologia da Universidade Estadual de Maringá. Rev ABENO. 2010;10(2):64-71.

14. Yamakami SA, Albiero ALM, Baesso ML, Fujimaki M, Terada RSS, Pascotto RC. Desenvolvendo o paradigma da integralidade no cuidado à saúde no PET-Saúde/UEM: relato de experiência: atuação de maneira articulada no cuidado integral à saúde da comunidade. Rev ABENO. 2014;14(1):4756.

15. Rocha NB, Silva MC, Silva IRG, Lolli LF, Fujimaki M, Alves RN. Percepções de aprendizagem sobre disciplina interprofissional em Odontologia. Rev ABENO. 2017;17(3):41-54.

16. Batista NA. Educação Interprofissional em Saúde: concepções e práticas. Cad FNEPAS.2012;2(1):25-28.

17. Toassi RFC, Lewgoy AMB. Práticas Integradas em Saúde I: uma experiência inovadora de integração intercurricular e interdisciplinar.

Interface

(Botucatu).2016;20(57):449-61.

18. Prado MLD, Velho MB, Espíndola DS, Sobrinho SH, Backes VMS. Arco de Charles Maguerez: refletindo estratégias de metodologia ativa na formação de profissionais de saúde. Esc Anna Nery. 2012;16(1):172-7.
19. Vieira VMO. Portfólio: uma proposta de avaliação como reconstrução do processo de aprendizagem. Psicol Esc Educ. 2002; 6(2):149-53.

20. Rangel JNM. O portfólio e a avaliação no ensino superior. Est Aval Educ. 2003; (28):145-60.

21. Frota MMA, Menezes LMBD, Alencar CH, Jorge LDS, Almeida MELD. O portfólio como estratégia facilitadora do processo de ensinoaprendizagem para a formação em odontologia: Adequação de metodologias de ensino utilizando o ambiente virtual de aprendizagem. Rev ABENO. 2011;11(1):23-8.

22. Bardin L. Análise de conteúdo. 3. ed. São Paulo: Ed. 70, 2004.

23. Cooper H, Spencer-Dawer E, McLean E. Beginning the process of teamwork: design, implementation and evaluation of an interprofessional education intervention for first year undergraduate students. J Interprof Care. 2005;19(5):492-508.

24. Barr H, Freeth D, Hammick M, Koppel I, Reeves S. Evaluations of interprofessional education: a United Kingdom review of health and social care. London: CAIPE/BERA. 2000.

25. Kruger LM. Método Tradicional e Método Construtivista de ensino no processo de aprendizagem: uma investigação com os acadêmicos da disciplina Contabilidade III do curso de Ciências Contábeis da Universidade Federal de Santa Catarina. ROC. 2013:219-70 26. Cotta RMM, Silva LS, Lopes LL, Gomes KO, Cotta FM, Lugarinho R, Mitre SM. Construção de portfólios coletivos em currículos tradicionais: uma proposta inovadora de ensino-aprendizagem. Ciênc Saúde Coletiva. 2012;17(3):787-96.

27. Rocha NHN, Bevilacqua PD, Barletto M. Metodologias participativas e educação permanente na formação de agentes 
comunitários/as de saúde. Rev Trab Educ Saúde. 2015;13(3):597-615.

28. Rocha JS, Dias GF, Campanha NH, Baldani MH. O uso da aprendizagem baseada em problemas na Odontologia: uma revisão crítica da literatura. Rev ABENO. 2016;16(1):25-38.

29. Marin MJS, Lima EFG, Paviotti AB, Matsuyama DT, Silva LKDD, Gonzalez C, et al. Aspectos das fortalezas e fragilidades no uso das metodologias ativas de aprendizagem. Rev Bras Educ Méd. 2010;34(1):13-20.

30. Santos NR. Desenvolvimento do SUS, rumos estratégicos e estratégias para visualização dos rumos. Ciênc Saúde Coletiva. 2007;12(2):429-35.

31. Cardoso JP, Vilela ABA, Souza NR, Vasconcelos CCO, Caricchio GMN. Formação interdisciplinar: efetivando propostas de promoção da saúde no SUS. Rev Bras Promoç Saúde. 2012;20(4):252-8.

32. Soares LT. As atuais políticas de saúde: os riscos do desmonte neoliberal. Rev Bras Enf. 2000;53:17-24.

33. Machado MFAS, Monteiro EMLM, Queiroz DT, Vieira NFC, Barroso MGT. Integralidade, formação de saúde, educação em saúde e as propostas do SUS: uma revisão conceitual. Ciênc Saúde Coletiva. 2007;12(2):335-42.

\section{Correspondência para:}

Najara Barbosa Rocha

e-mail: najara.rocha@gmail.com

Av. Mandacaru, 1550. Bloco S08

87083-170 Maringá/PR 